\title{
Correção cirúrgica dos aneurismas da aorta: novo dispositivo que transforma qualquer tipo de prótese em prótese intraluminal
}

Rodrigo de Castro BERNARDES ${ }^{*}$, Fernando Antônio Roquette REIS FILHO*, Atena Cipriano CASTRO*, Walter RABELO*, Marcos Antônio MARINO*, Roberto Luiz MARINO*, Raul Correa RABELO

RBCCV 44205-231

BERNARDES, R. C.; REIS FILHO, F. A. R.; CASTRO, A. C.; RABELO, W.; MARINO, M. A.; MARINO, R. L.; RABELO, R. C. - Correção cirúrgica dos aneurismas da aorta: novo dispositivo que transforma qualquer tipo de prótese em prótese intraluminal. Rev. Bras. Cir. Cardiovascular, 9(1):54-59, 1994.

RESUMO: Os autores propōem o tratamento dos aneurismas da aorta com prótese intraluminal. É usado um novo dispositivo que transforma prótese de tipo, tamanho e diâmetro diferentes em prótese intraluminal, excluindo qualquer tipo de sutura para a fixação, e usando apenas uma ligadura circunferencial extravascular. Entre maio de 1989 e janeiro de 1993, 22 pacientes foram submetidos a aneurismectomia da aorta usando esta técnica: 12 pacientes com dissecção aguda da aorta tipo I e ll de DeBAKEY, 1 paciente com dissecção aguda da aorta tipo III de DeBAKEY, 2 pacientes com aneurisma da aorta descendente, 2 pacientes com aneurisma da aorta toracoabdominal e 5 pacientes com aneurisma da aorta abdominal. Três pacientes evoluíram para - óbito no pós-operatório por acidente vascular cerebral, trombose mesentérica e infecção pulmonar, respectivamente. Os pacientes sobreviventes estão sendo seguidos trimestralmente em ambulatório e encontram-se assintomáticos. O uso do anel rígido sulcado de Delrin (R) simplifica a técnica cirúrgica, reduzindo o tempo de circulaçāo extracorpórea e de parada cardiaca, dispensando o uso de hipotermia profunda e reduzindo o sangramento. Permite ainda o seu uso em qualquer local da aorta de maneira rápida, fácil e a baixo custo.

DESCRITORES: aneurismas de aorta, prótese intraluminal, cirurgia.

\section{INTRODUÇÃO}

O tratamento cirúrgico dos aneurismas da aorta já está consagrado mundialmente por apresentar menor mortalidade que o tratamento clínico 8, 12 . Entretanto, apesar dos avanços da cirurgia cardiovascular moderna, os aneurismas da aorta ainda causam grande morbidade e mortalidade 2, 7,10.

A mortalidade cirúrgica é freqüentemente resultado de hemorragia nas linhas de sutura, complicações de transfusão sangũínea maciça, tempo prolongado de circulação extracorpórea com hipotermia profunda e parada circulatória total 11,12 .
O conceito de prótese intraluminal com ligadura circunferencial foi sugerido primeiramente por CARREL ${ }^{4}$ em 1912 e adotado posteriormente por HUFNAGEL 7 .

BERGER et alii ${ }^{2}$ propuzeram o tratamento da dissecção aguda da aorta com prótese intraluminal, na expectativa de realizar uma cirurgia rápida, com anastomose hemostática.

A prótese intraluminal foi comercializada em 1980 e no início apresentou sérias dificuldades técnicas, devido a seu comprimento fixo industrializado, além dos casos de migração e sangramento cirúrgico através dos pontos de fixação 6,8 .

Trabalho realizado no Instituto do Coração do Hospital Madre Tereza, Belo Horizonte, MG. Brasil.

Recebido para publicação em janeiro de 1994.

- Do Instituto do Coração do Hospital Madre Tereza.

Endereço para correspondência: Rodrigo Bernardes. Av. Raja Gabaglia, 1002, Gutierrez. 30380-090 Belo Horizonte, MG. Brasil. 
BERNARDES, R. C.; REIS FILHO, F. A. R.; CASTRO, A. C.; RABELO, W.; MARINO, M. A.; MARINO, R. L.; RABELO, R. C. Correção cirúrgica dos aneurismas da aorta: novo dispositivo que transforma qualquer tipo de prótese em prótese intraluminal. Rev. Bras. Cir. Cardiovascular, 9(1):54-59, 1994.

GRAVER \& TYRAS 6 propuzeram o uso de prótese intraluminal de comprimento variável.

Apesar do nome prótese intraluminal sem sutura, este tipo de prótese exige alguns pontos para fixação do anel semi-rígido.

Desenvolvemos em nosso serviço um anel rígido, sulcado de Delrin (R), que serve de suporte para transformar prótese vascular em prótese intraluminal, de qualquer tamanho ou comprimento, dispensando sutura de fixação e usando apenas ligadura circunferencial extravascular.

\section{CASUISTICA E MÉTODOS}

Em 1988, desenvolvemos o anel rígido sulcado de Delrin (R) com a intenção de suportar próteses vasculares, transformando-as em prótese intraluminal (Figuras 1, 2, 3). Após experimentos em aorta de cães, constatamos que este anel possibilitava uma anastomose muito rápida, fácil e hemostática, usando apenas uma ligadura circunferencial extravascular.

Entre maio de 1989 e janeiro de 1993, 22 pacientes foram submetidos a aneurismectomia da aorta, utilizando a técnica ilustrada. Todos os pacientes eram do sexo masculino, com idades variando de 26 a 78 anos (média de 63 anos); 12 pacientes apresentavam DISSECÇÃO AGUDA DA AORTA TIPO I e II de DeBAKEY, 1 paciente DISSECÇÃO AGUDA DE AORTA TIPO III de DeBAKEY, 2 pacientes ANEURISMA DE AORTA DESCENDENTE, 2 pacientes ANEURISMA DE AORTA TORACOABDOMINAL e 5 pacientes ANEURISMA DE AORTA ABDOMINAL (Quadro 1).

Todos os pacientes foram submetidos a cateterização cardíaca com aortografia, ecodopplercardiografia e tomografia computadorizada para diagnóstico.

O tratamento cirúrgico foi realizado logo após o diagnóstico.

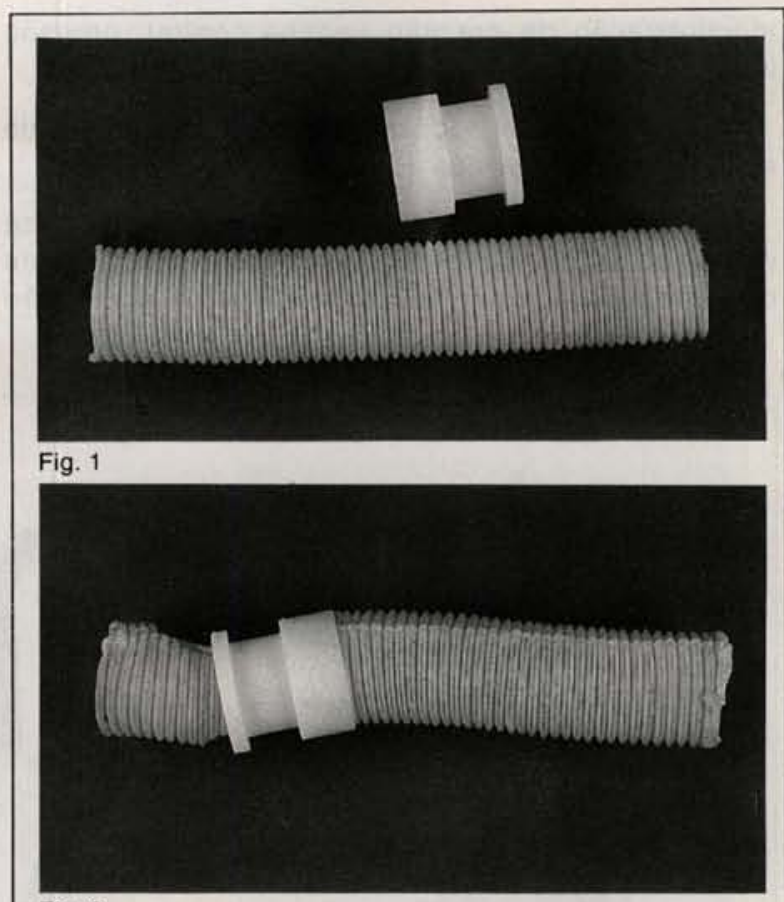

Fig. 2

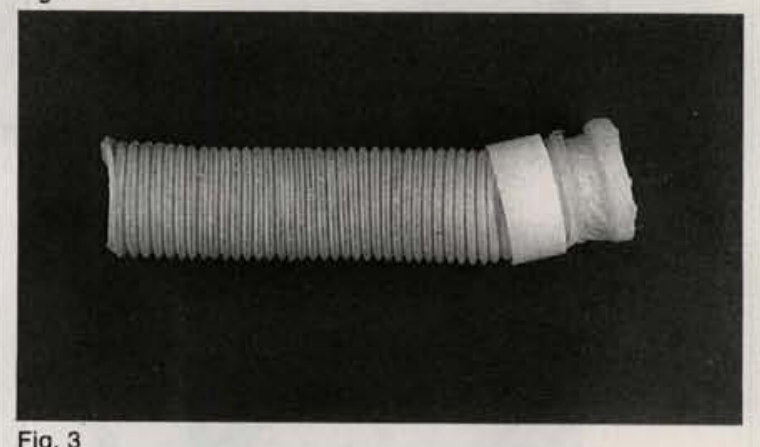

Figuras 1, 2 e 3 -Sequência de montagem do anel rígido de Delrin $(R)$ em prótese vascular tubular reta, transformando-a em endoprótese.

\section{Técnica cirúrgica}

Todos os pacientes foram operados sob anestesia geral, monitorização eletrocardiográfica,

QUADRO 1

DADOS CLÍNICOS

\begin{tabular}{lcc}
\hline DIAGNÓSTICO & NoPACIENTES & SOBREVIVENTES \\
\hline Dissecção aguda Ao tipo I e II & 12 & $10(80 \%)$ \\
Dissecção aguda Ao tipo III & 1 & $1(100 \%)$ \\
Aneurisma de Ao descendente & 2 & $2(100 \%)$ \\
Aneurisma de Ao tóraco-abdominal & 2 & $1(50 \%)$ \\
Aneurisma de Ao abdominal & 5 & $5(100 \%)$ \\
\hline TOTAL & 22 & $19(86,4 \%)$ \\
\hline
\end{tabular}


BERNARDES, R. C.; REIS FILHO, F. A. R.; CASTRO, A. C.; RABELO, W.; MARINO, M. A.; MARINO, R. L.; RABELO, R. C. -

Correção cirúrgica dos aneurismas da aorta: novo dispositivo que transforma qualquer tipo de prótese em prótese intraluminal. Rev. Bras. Cir. Cardiovascular, 9(1):54-59, 1994.

monitorização da pressão venosa central, pressão intra-arterial e controle do volume urinário.

O princípio da técnica cirúrgica está ilustrado esquematicamente nas Figuras $4,5,6,7$ e 8 .

Os pacientes submetidos a correção cirúrgica de dissecção da aorta tipo I e II de DeBAKEY, foram operados por esternotomia mediana e circulação extracorpórea instalada por canulação do átrio direito e artéria femoral, em normotermia. Após pinçamento da aorta, a proteção miocárdica foi obtida através de cardioplegia sangüínea retrógrada contínua, normotérmica. Procedeu-se, então, a incisão longitudinal em aorta ascendente, sendo inspecionada a sua luz, procurando-se identificar o ponto de rotura da íntima.

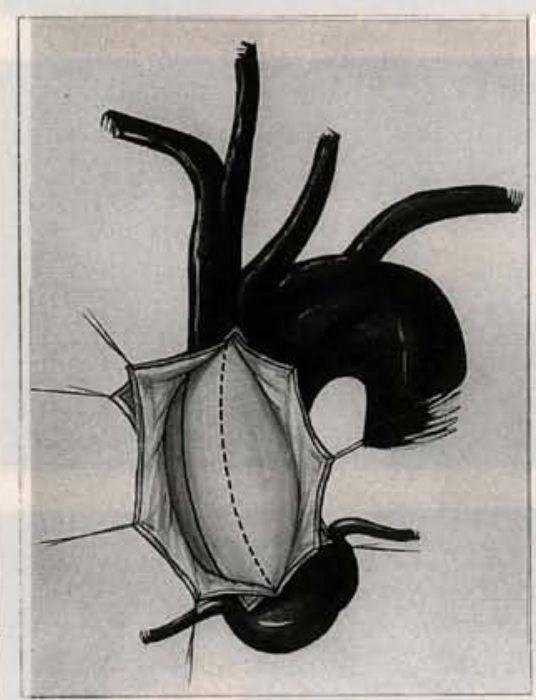

Fig. 4

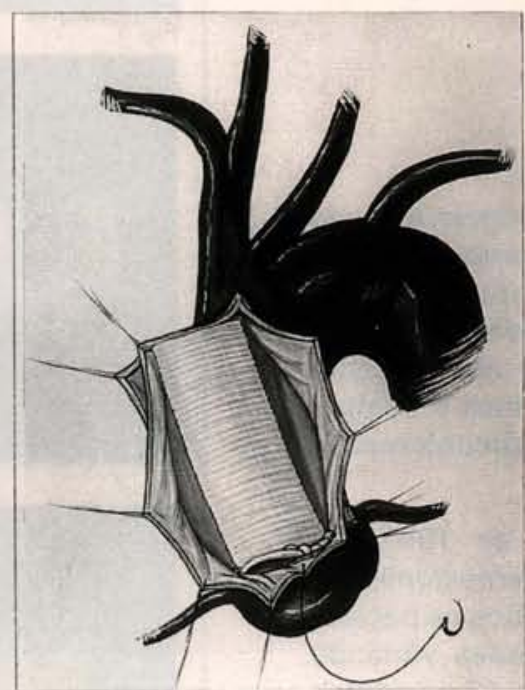

Fig. 5

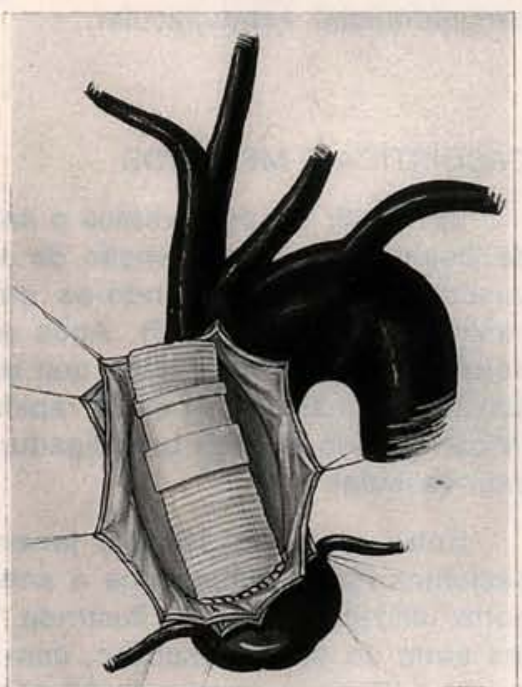

Fig. 6
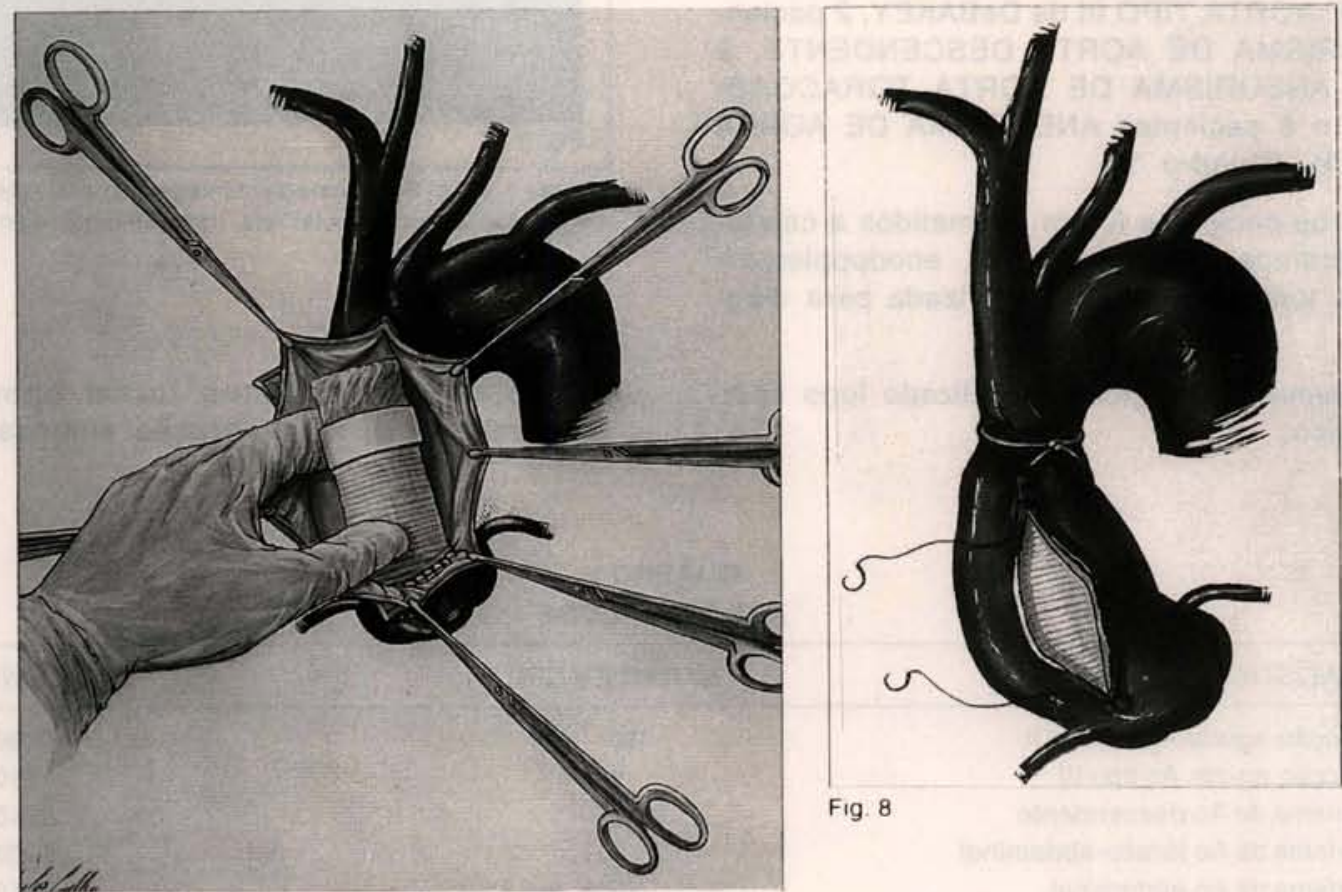

Fig. 8

Fig. 7

Figuras 4, 5, 6, 7 e 8 - Seqũência da técnica cirúrgica empregada, usando o anel rígido sulcado de Delrin (R) 
BERNARDES, R. C.; REIS FILHO, F. A. R.; CASTRO, A. C.; RABELO, W.; MARINO, M. A.; MARINO, R. L.; RABELO, R. C. Correção cirúrgica dos aneurismas da aorta: novo dispositivo que transforma qualquer tipo de prótese em prótese intraluminal. Rev. Bras. Cir. Cardiovascular, 9(1):54-59, 1994.

Os pacientes que apresentavam insuficiência da valva aórtica ( 5 casos) foram tratados em prótese de pericárdio bovino corrugado Labcor (R) acima dos óstios coronarianos, de modo a suspender a valva aórtica. Passamos, então, a prótese tubular por dentro do anel rígido sulcado e a invertemos sobre o mesmo, "vestindo-o". Procedeu-se, então, a curta parada circulatória total, nunca superior a 60 segundos, tempo suficiente para posicionar a prótese dentro da luz verdadeira da aorta, seguindo-se firme ligadura extravascular com fio de Poliester trançado 5 .

Os pacientes que não apresentavam insuficiência da valva aórtica ( 7 casos) foram tratados empregando o anel rígido nas duas extremidades da prótese, procedendo-se à primeira ligadura da endoprótese logo acima dos óstios coronarianos, e a outra conforme técnica já descrita.

Os pacientes submetidos a correção cirúrgica de dissecção aguda da aorta tipo III de DeBAKEY, aneurisma da aorta descendente e aneurisma de aorta toracoabdominal (5 casos), foram operados sem circulação extracorpórea e sem heparinização sistêmica. Empregou-se pinçamento da aorta e aortotomia longitudinal. Uma prótese de Dacron précoagulada com colágeno foi passada por dentro do anel rígido e invertida sobre o mesmo em suas extremidades distal e proximal. Após ser posicionada na luz verdadeira da aorta, seguiu-se firme ligadura circunferencial extravascular sobre os sulcos dos anéis com fios de Poliester trançado 5 .

Quando foi necessária a anastomose de ramos espinhais e viscerais, empregamos a técnica de inclusão de CRAWFORD et alii ${ }^{5}$.

Os pacientes portadores de aneurisma da aorta abdominal ( 5 casos) foram submetidos a heparinização sistêmica para pinçamento da aorta e abertura do saco aneurismático. Uma prótese bifurcada de pericárdio bovino corrugado Labcor (R) foi passada por dentro do anel rígido e invertida sobre o mesmo, "vestindo-o". Após ser posicionada na luz da aorta, logo abaixo das artérias renais, procedeu-se a firme ligadura com fio de Poliester trançado 5 sobre o sulco do anel. As anastomoses distais foram realizadas de modo habitual (Figuras 9 e 10)

\section{RESULTADOS}

Os tempos de circulação extracorpórea e de pinçamento da aorta nos pacientes portadores de dissecção da aorta tipo I e II foram reduzidos substancialmente. Em nenhum paciente usamos hipotermia profunda.

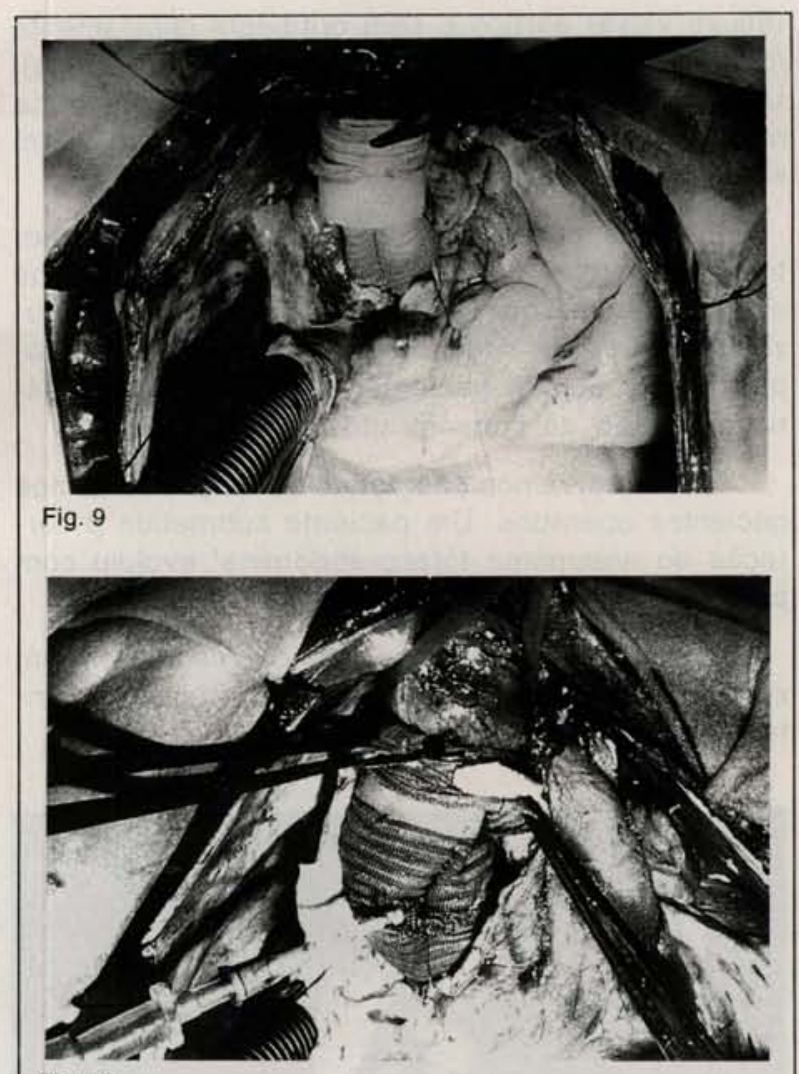

Fig. 10

Figuras. 9 e 10 - Sequiência do aspecto cirúrgico do implante da endoprótese.

O tempo de parada circulatória total foi menor que 60 segundos em todos os casos, tempo suficiente para a introdução e fixação da endoprótese.

O sangramento cirúrgico e a perda sangüínea no pós-operátorio foram mínimos e nenhum dos pacientes recebeu transfusão de concentrado de plaquetas no pós-operatório, ou foi reoperado para revisão da hemostasia.

Ocorreram $3(13,63 \%)$ óbitos no período de pós-operatório. O $1^{\circ}$ paciente faleceu no $20^{\circ}$ dia de pós-operatório após correção de dissecção aguda de aorta tipo I de DeBAKEY, por acidente vascular cerebral isquêmico diagnosticado no período préoperatório; o $2^{\circ}$ paciente faleceu no $1^{\circ}$ dia de pósoperatório após correção de dissecção aguda da aorta tipo I de DeBAKEY por trombose mesentérica; - $3^{\circ}$ paciente faleceu no $26^{2}$ dia de pós-operatório após aneurismectomia da aorta tóraco-abdominal com infecção pulmonar e falência de múltiplos órgãos.

Os pacientes com dissecção aguda da aorta sobreviventes foram submetidos a estudo hemodinâmico, que mostrou correção da dissecção, sem 
BERNARDES, R. C.; REIS FILHO, F. A. R.; CASTRO, A. C.; RABELO, W.; MARINO, M. A.; MARINO, R. L.; RABELO, R. C. Correção cirúrgica dos aneurismas da aorta: novo dispositivo que transforma qualquer tipo de prótese em prótese intraluminal. Rev. Bras. Cir. Cardiovascular, 9(1):54-59, 1994.

refluxo valvar aórtico e sem gradiente clinicamente importante ao nível da prótese (média de $20 \mathrm{mmHg}$ ). Um dos pacientes foi submetido a ressonância magnética que demonstrou aorta normal, sem estenoses (Figura 11).

Para os pacientes submetidos a aneurismectomia da aorta torácica e abdominal, os tempos cirúrgico e de isquemia visceral foram muito reduzidos com perda sangüínea mínima (média de reposição de $600 \mathrm{ml} /$ paciente), não sendo necessária a infusão de concentrado de plaquetas.

Não observamos alterações da função renal nos pacientes operados. Um paciente submetido a correção de aneurisma tóraco-abdominal evoluiu com paraplegia no pós-operatório.

O seguimento pós-operatório dos pacientes vem sendo feito em regime ambulatorial, trimestralmente, estando todos assintomáticos.

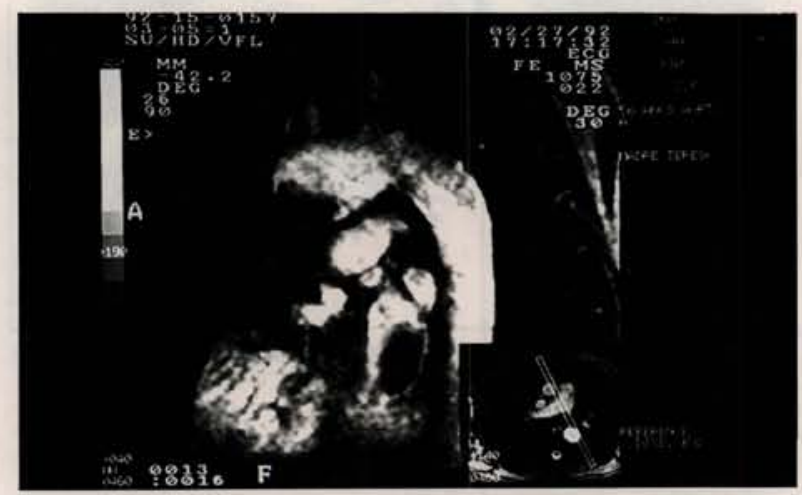

Figura 11 - Imagem de ressonância magnética mostrando endoprótese em aorta ascendente.

\section{COMENTÁRIOS}

Frente a um aneurisma da aorta, o cirurgião necessita estar preparado para reparar ou trocar toda a aorta, através de uma variedade de técnicas, pois a precisa avaliação anatômica no pré-operatório nem sempre é possível ${ }^{6}$. Segundo OLINGER et alii ${ }^{9}$ a vantagem de se intervir na origem da dissecção em aorta ascendente está na prevenção de dissecção retrógrada e hemorragia intrapericárdica, correção da insuficiência aórtica e prevenção de oclusão dos óstios coronarianos.

Diversos autores 10,11 mostraram que a maioria dos pacientes submetida a tratamento cirúrgico de dissecção em aorta ascendente apresenta falso lume, assintomático persistente; os pacientes são tratados clinicamente com betabloqueadores, anti-hipertensivos e em observação clínica.

GRAVER \& TYRAS ${ }^{6}$ acreditam que o tratamento cirúrgico, com prótese intraluminal e suspensão da valva aórtica, usando parada circulatória, tem muitas vantagens sobre a técnica de sutura direta.

O anel rígido, sulcado de Delrin (R) por nós desenvolvido tem a vantagem de transformar prótese, de qualquer tamanho ou comprimento, em prótese intraluminal, completamente sem sutura, usando apenas ligadura circunferencial extravascular, podendo ser usada em qualquer ponto da aorta, (ascendente, descendente ou abdominal), de maneira rápida, fácil, sem riscos maiores de sangramento e a muito baixo custo.

Em nossos pacientes operados por esta técnica, não foi necessária a hipotermia profunda, não ocorreu qualquer caso de sangramento e nenhum paciente necessitou do uso de concentrado de plaquetas no pós-operatório.

Não observamos redução acentuada do calibre da aorta ou aparecimento de gradiente significativo transanular nos sobreviventes, e não ocorreu nenhum caso de embolia arterial no pós-operatório.

Concluindo, entendemos que o uso da prótese intraluminal no tratamento dos aneurismas da aorta, em casos selecionados, simplifica muito a técnica, diminuindo o tempo cirúrgico, o sangramento e naturalmente a morbidade e a mortalidade. $O \mathrm{em}$ prego do anel rígido, sulcado, de Delrin (R), estende a técnica a todos os segmentos da aorta torácica ou abdominal. 
BERNARDES, R. C.; REIS FILHO, F. A. R.; CASTRO, A. C.; RABELO, W.; MARINO, M. A.; MARINO, R. L.; RABELO, R. C. Correção cirúrgica dos aneurismas da aorta: novo dispositivo que transforma qualquer tipo de prótese em prótese intraluminal. Rev. Bras. Cir. Cardiovascular, 9(1):54-59, 1994.

\section{RBCCV 44205-231}

BERNARDES, R. C.; REIS FILHO, F. A. R.; CASTRO, A. C.; RABELO, W.; MARINO, M. A.; MARINO, A. L.; RABELO, R. C. - Surgical correction of aortic aneurysms: a new device for conversion of any graft of intraluminal one. Rev. Bras. Cir. Cardiovasc., 9 (1): 54-59, 1994.

ABSTRACT: The authors propose the treatment of aortic aneurysms by using an intraluminal graft. A new device which transforms any kind of graft of different sizes and diameters into an intraluminal one is described. This new device just needs an extra-vascular circunferential ligature and does not require any fixation suture. The use of the rigid, sulcated DELRIN (R) ring simplifies the technique shortening the duration of the surgery, reducing surgical bleeding and dispensing the use of profound hypothermia and platelet transfusion. Between May 1989 and January 1993, 22 patients were submited to aneurysmectomy of the aorta using this technique. Twelve patients exhibited acute aortic dissection (type I and II DeBakey), one DeBakey type III acute aortic dissection, two presented descending aortic aneurysm, two cases were of thoraco-abdominal aortic aneurysm and five aneurysms of abdominal aorta. Three patients died in the imediate post-operative period from cerebrovascular accident, mesenteric thrombosis and pulmonary infection. The survivors have been followed up as outpatients.

DESCRIPTORS: aneurysms, aortic, intraluminal graft, surgery.

\section{REFERÊNCIAS BIBLIOGRÁFICAS}

1 ABLASA, S. G.; GHOSH, S. C.; GRANA, V. P. - Use of the ringed intraluminal graft in the surgical treatment of dissecting aneurysms of the toracic aorta. $J$. Thorac. Cardiovasc. Surg., 76: 390-396, 1978.

2 BERGER, R. L.; ROMERO, L.; CHAUDHRY, A. G.; DOBNIK, D. B. - Graft replacement of the thoracic aorta with a sutureless technique. Ann. Thorac. Surg., 35: 231-239, 1983.

CABROL, C.; PRAVIE, A.; MESNILDREY, P. - Longterm results with total replacement of the ascending aorta and reimplantation of the coronary arteries. J. Thorac. Cardiovasc. Surg., 91: 17-25, 1986.

CARREL, A. - Results of the permanent intubation of the thoracic aorta. Surg. Gynecol. Obstet., 15:245-248, 1912.

CRAWFORD, E. S.; COSELLI, J. S.; SVENSSON, L. G.; SAFI, H. J.; HESS, K. R. - Diffuse aneurysmal disease (chronic aortic dissection, Marfan, and mega aortic syndrome) and multiple aneurysm treatment by subtotal and total aortic replacement emphasizing the elephant trunk operation. Ann. Surg., 211: 521 . $527,1990$.
GRAVER, L. M. \& TYRAS, D. H. - Use of the variablelength intraluminal sutureless graft. Ann. Thorac. Surg., 50: 624-630, 1990.

HUFNAGEL, C. A. - Aortic plastic valvular prosthesis. Bull. Georgetown. Univ. Med. Ctr., 4: 128-199, 1951.

MILLER, D. C.; STINSON, E. B.; OYER, P. E. - Operative treatment of aortic dissections-experience with 125 patients over a sixteen-year period. J. Thorac. Cardiovasc. Surg., 78: 365-382, 1979.

9 OLINGER, G. A.; SCHWEIGER, R. N.; GALBRAITH, T. A. - Primary repair of acute ascending aortic dissection. Ann. Thorac. Surg., 44: 389-393, 1987.

10 THOMAS, C. S.; ALFORD, W. C.; BURRUS, G. R.; FRIST, R. A.; STONEY, W. S. - The effectiveness of surgical treatment of acute aortic dissection. Ann. Thorac. Surg., 26: 42-49, 1978.

11 TURLEY, K.; ULLYOT, D. J.; GODWIN, J. D. - Repair of dissection of the thoracic aorta: evaluation of false lumen utilizing computed tomography. J. Thorac. Cardiovasc. Surg., 81: 61-68, 1981.

12 WHEAT, M. J. - Acute dissecting aneurysms of the aorta: diagnosis and treatment. Am. Heart J., 99: 373-387, 1980 . 